\title{
不同邻菲洛琳衍生物作为中性配体的三元铺 配合物电致发光性质研究"
}

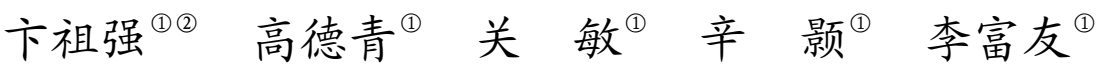 \\ 王科志 ${ }^{\circledR}$ 金林培 ${ }^{\circledR}$ 黄春辉 ${ }^{\mathbb{N}^{* *}}$
}

(1)北京大学稀土材料化学与应用国家重点实验室，北京 100871; (2)北京师范大学化学系，北京 100875)

\begin{abstract}
摘要 合成了三个可真空蒸镀成膜的三元销配合物 $\operatorname{Eu}(\mathrm{DBM})_{3} \mathrm{LN}$ (DBM 为二苯甲酰甲烷阴离 子, $\mathrm{LN}$ 代表不同邻菲洛琳衍生物), 并对其电致发光性质进行了研究. 在配合物 $\mathrm{Eu}(\mathrm{DBM})_{3} \mathrm{~L} 3\{\mathrm{~L} 3$ =2-苯基-3-[3-(咔唑-9)丙基]咪唑 [4, 5-f]1, 10-邻菲洛琳 $\}$ 中，引入邻菲洛琳和咔唑基团以期分别提 高材料的电子和空穴传输性能. 研究结果表明, 对中性配体的有效修饰能够改善材料的热稳定 性、载流子传输性和光致发光性质, 从而显著提高其电致发光性能. 双层器件 ITO/TPD (40 nm)/ $\mathrm{Eu}(\mathrm{DBM})_{3} \mathrm{~L} 3(80 \mathrm{~nm}) / \mathrm{Mg}: \mathrm{Ag}(200 \mathrm{~nm}) / \mathrm{Ag}(100 \mathrm{~nm})$ 能够发出销配合物的特征荧光, 启动电压为 $8 \mathrm{~V}$, 最大亮度达 $561 \mathrm{~cd} / \mathrm{m}^{2}(16 \mathrm{~V})$. 四层器件 ITO/TPD $(50 \mathrm{~nm}) /\left[\mathrm{Eu}(\mathrm{DBM})_{3} \mathrm{~L} 3(5 \mathrm{~nm}): \mathrm{BCP}(5 \mathrm{~nm})\right]_{4} /$ $\mathrm{BCP}(20 \mathrm{~nm}) / \mathrm{AlQ}(10 \mathrm{~nm}) \mathrm{Mg}_{0.9} \mathrm{Ag}_{0.1}(110 \mathrm{~nm}) / \mathrm{Ag}(100 \mathrm{~nm})$ 的最大亮度达 $1419 \mathrm{~cd} / \mathrm{m}^{2}(18 \mathrm{~V})$, 也为钴配 合物的特征发射.
\end{abstract}

\section{关键词 二苯甲酰甲烷 电致发光 稀土 含 $\mathbf{N}$ 配体}

稀土配合物具有独特的光学性质, 如色纯度高、 理论上荧光量子效率接近 $100 \%$, 是理想的电致发光 材料, 十几年来一直是研究热点之一, 发达国家都有 相应的科学小组投入了这方面的工作 ${ }^{[13]}$. 但目前文 献报道的稀土电致发光结果还不尽如人意，与其他 电致发光材料相比, 亮度不高、效率低.

一般认为, 作为一个优异的电致发光材料, 稀土
配合物应同时兼具良好的光致发光性能、热稳定性、 成膜性以及好的载流子传输性能. 许多稀土配合物 具有高的光致发光效率，但很少有同时满足上述条 件的. $\mathrm{Eu}(\mathrm{DBM})_{3} \mathrm{Phen}$ 具有良好光致发光性质, 但是由 于其载流子传输性能较差, 用它作为电致发光 材 料所制作的器件 ITO/CuPc $(5 \mathrm{~nm}) / \alpha-\mathrm{NPD}(35 \mathrm{~nm}) /$ $\mathrm{Eu}(\mathrm{DBM})_{3} \mathrm{Phen}(20 \mathrm{~nm}) / \mathrm{BCP}(15 \mathrm{~nm}) / \mathrm{AlQ}(25 \mathrm{~nm}) / \mathrm{LiF} /$ 
$\mathrm{A} 1$ 最大亮度仅为 $50 \mathrm{~cd} / \mathrm{m}^{2}(15 \mathrm{~V})^{[2]}$. 当将它掺杂在具 有能隙较宽的电荷传输材料如 $N, N^{\prime}$-二苯基 $-N, N^{\prime}$ 二 二 (1-萗基)-1， $1^{\prime}$-二苯基-4，4'-二胺( $\alpha$-NPD)或 2-(4-联苯 基)-5-(4-叔丁基苯基)-1, 3, 4-噁二唑(PBD)时，可以提 高其电致发光性能. $\mathrm{Eu}(\mathrm{DBM})_{3} \mathrm{Phen}$ 掺杂在空穴传输 材料 $\alpha$-NPD中所制得器件ITO/CuPc $(5 \mathrm{~nm}) / \alpha-\mathrm{NPD}(35$ nm)/ $\alpha-N P D: \operatorname{Eu}(\mathrm{DBM})_{3} \operatorname{Phen}(20 \%: 80 \%)(20 \mathrm{~nm}) / \mathrm{BCP}$ $(15 \mathrm{~nm}) / \mathrm{AlQ}(25 \mathrm{~nm}) / \mathrm{LiF} / \mathrm{Al}$ 的最大亮度在 $15 \mathrm{~V}$ 达 200 $\mathrm{cd} / \mathrm{m}^{2[2]}$. 将 $\mathrm{Eu}(\mathrm{DBM})_{3}$ Phen掺杂在电子传输材料 $\mathrm{PBD}$ 中所制得器件 ITO/TPD $(40 \mathrm{~nm}) / \mathrm{Eu}(\mathrm{DBM})_{3}$ Phen : $\operatorname{PBD}(1: 3, \mathrm{~mol}: \mathrm{mol})(30 \mathrm{~nm}) / \mathrm{AlQ}(30 \mathrm{~nm}) / \mathrm{Mg}: \mathrm{Ag}$ 的最大亮度在 $16 \mathrm{~V}$ 时达 $460 \mathrm{~cd} / \mathrm{m}^{2[4]}$. 但是, 共蒸掺杂 技术也有缺点——如混和材料在器件使用时易分相, 共蒸蒸镀条件难以控制等. 另一有效的改善载流子 传输性能的方法是对稀土配合物中的配体进行合理 修饰，引入功能基团，优化材料性能. 2001 年, Zheng 研究小组 ${ }^{[7]}$ 将具有电子传输性能的基团噁唑连接到 乙酰丙酮中制备了配体 3-(5-苯基-1，3，4-噁二唑- 2基)-2，4-戊二酮 (POP), 并将其与铽配位形成配合物 $\mathrm{Tb}(\mathrm{POP})_{3}\left(\mathrm{H}_{2} \mathrm{O}\right)_{2}$. 器件 ITO/PVK : PBD : $\mathrm{Tb}(\mathrm{POP})_{3}$ $\left(\mathrm{H}_{2} \mathrm{O}\right)_{2} / \mathrm{Alpop} / \mathrm{CsF} / \mathrm{Al}$ 在 $15 \mathrm{~V}$ 时亮度为 $100 \mathrm{~cd} / \mathrm{m}^{2}$, 外 量子效率达 $1.1 \%(0.4 \mathrm{~cd} / \mathrm{A})$. 因此, 进一步探索合成 满足优异电致发光条件的材料非常重要.

为了改善材料的载流子传输性能，本工作通过 分子修饰，设计、合成了三个邻菲洛琳衍生物(图 1) 及其相应的铕混配配合物, 比较了不同的中性配体 对材料性能的影响。研究结果表明，配合物 $\mathrm{Eu}(\mathrm{DBM})_{3} \mathrm{~L} 3$ 由于引入了邻菲洛琳和咔唑基团, 分别 有效提高了材料的电子和空穴传输性能，从而显著 改善了其电致发光性能.

\section{1 实验部分}

\section{1 器件制作}

导电玻璃(ITO, $150 \mathrm{~nm}$ 厚氧化铟锡, 方块电阻为 $15 \Omega / \mathrm{sq}$ )由中国南方玻璃有限公司(深圳)提供，使用 前按文献方法清洗. 在高真空 $\left(8 \times 10^{-4} \mathrm{~Pa}\right)$ 条件下用钼 舟将有机层依次蒸镀到导电玻璃ITO上，再镀上镁银 合金 $\left(\mathrm{Mg}_{0.9} \mathrm{Ag}_{0.1}\right)$ 做阴极，有机层蒸镀的速率为 $0.1 \sim 0.2 \mathrm{~nm} / \mathrm{s}$, 金属层为 $0.1 \sim 1.0 \mathrm{~nm} / \mathrm{s}$. 北京仪器厂生 产的IL-100 型膜厚速率监控仪对膜厚及其蒸发速率 实施监控, 并用Dektak台阶仪校正 ${ }^{[6]}$. 器件发光面积 为 $20 \mathrm{~mm}^{2}$.

\section{2 仪器及实验方法}

紫外可见光谱在Shimadzu UV-2400 型紫外分光 光度计上测定, ${ }^{1} \mathrm{H}$ NMR谱在Varian $400 \mathrm{MHz}$ 核磁共 振仪上记录，四甲基硅烷作内标，热重和差热分析在 LCT-1 型DSC分析仪上测定, 测定条件为大气氛下, 升温速度 $10^{\circ} \mathrm{C} / \mathrm{min}$. 器件亮度-电压-电流曲线由计算 机控制的Keithley 2400 源表测定，亮度由ST-86LA型 亮度计的硅二极管校正. 苂光光谱(光致发光和电致 发光)在日立F-4500 苂光分光光度计上测定，苂光量 子效率测定以 $\mathrm{EuCl}_{3} \cdot 6 \mathrm{H}_{2} \mathrm{O}$ 为标准 $\left(\varphi_{\mathrm{std}}=0.73 \%{ }^{[14]}\right)$, 参 照文献[15]，根据下列公式计算而得

$$
\varphi_{\mathrm{s}}=\varphi_{\mathrm{std}} \frac{\mathrm{A}_{\mathrm{std}}}{\mathrm{A}_{\mathrm{s}}} \frac{\mathrm{I}_{\mathrm{s}}}{\mathrm{I}_{\mathrm{std}}} \frac{\eta_{s}^{2}}{\eta_{\mathrm{std}}^{2}}
$$

其中, $\phi$ 为量子效率, $I$ 为荧光强度, $A$ 为吸光度 $(<0.1)$, $n$ 为折光率, $x$ 表示样品的数值, sta 表示标准的数值. 配合物为浓度在 $2 \sim 4 \times 10^{-4} \mathrm{~mol} / \mathrm{L}$ 的无水四氢呋喃溶 液.

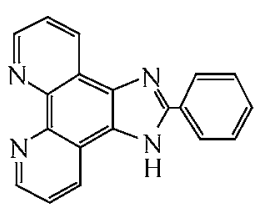

L) 1

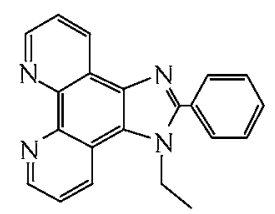

L2

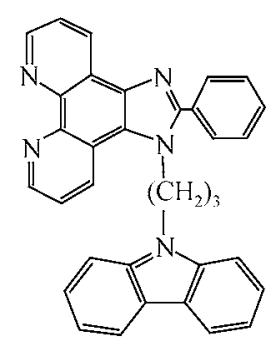

L3

图 1 配体 $\mathrm{L} N$ 的分子结构 $(N=1 \sim 3)$ 


\section{3 材料}

（i ）八羟基喹啉铝(AlQ)、 $N, N^{\prime}-$ 二苯基 $-N, N^{\prime}-$ 二 (1-菜基)-1, $1^{\prime}$-二苯基-4, 4'-二胺( $\alpha$-NPD)、2-(4-联苯 基)-5-(4-叔丁基苯基)-1, 3, 4-噁二唑(PBD)、2, 5-二苯 基-1, 3, 4-惡二唑(OXD)、 $N, N^{\prime}$-二苯基- $N, N^{\prime}$-二(3-甲 基苯基)-1, 1'-二苯基-4, 4'-二胺(TPD)、2, 9-二甲基-4, 7-二苯基-1, 10-菲洛琳(BCP)由美国 Aldrich 公司购买, 其余均为国产试剂. $N, N^{\prime}$-二甲基甲酰胺、正已烷和四 氢呋喃分别通过 $\mathrm{P}_{2} \mathrm{O}_{5}, \mathrm{CaH}_{2}$ 和金属钠在惰性气氛下 回流进行脱水处理. 配体及相应配合物的合成路线 如图 2 所示.

(ii) 2-苯基咪唑[4,5-f]1, 10-菲洛琳(L1) 仿文 献[16]方法, 用 1，10-菲洛琳-5，6-二酮与苯甲醛缩合 而得. 粗产品用 $N, N^{\prime}$-二甲基甲酰胺、二氯甲烷和乙醚 混合溶剂重结晶. 产率 75\%，元素分析测定值(\%): C, 73.17, $\mathrm{H}, 5.35, \mathrm{~N}, 15.97 ; \mathrm{C}_{19} \mathrm{H}_{12} \mathrm{~N}_{4} \mathrm{C}_{2} \mathrm{H}_{5} \mathrm{OH}$ 计算值(\%): C, 73.66, H, 5.30, N, 16.36.

(iii) 三(二苯甲酰甲烷基))\{2-苯基咪唑 $[4,5-f] 1$, 10 - 菲洛琳 $\}$ 合铕 $(\mathrm{III})\left[\mathrm{Eu}(\mathrm{DBM})_{3} \mathbf{L 1}\right]$ 将等摩尔的 $\mathrm{Eu}(\mathrm{DBM})_{3} \cdot 2 \mathrm{H}_{2} \mathrm{O}(0.85 \mathrm{~g}, 1.0 \mathrm{~m} \mathrm{~mol})$ 和配体 $\mathrm{L} 1(0.34 \mathrm{~g}$, $1.0 \mathrm{mmol}$ )溶于 $25 \mathrm{~mL} \mathrm{THF}$, 回流 $2 \mathrm{~h}$ 后, 加入乙醇, 析出黄色固体，抽滤，固体用少量乙醇洗涤，烘干. 产率 82\%, 元素分析测定值(\%): C, 66.21, H, 4.34, N, 4.51; $\mathrm{C}_{64} \mathrm{H}_{45} \mathrm{~N}_{4} \mathrm{O}_{6} \mathrm{Eu} \cdot 2 \mathrm{H}_{2} \mathrm{O}$ 计算值(\%): C, 66.61, H, $4.28, \mathrm{~N}, 4.86$.

(iv) 3-乙基-2-苯基咪唑 $[4,5$-f]1，10-菲洛琳(L2)

氮气氛保护下, 用无水正已烷洗去 $0.288 \mathrm{~g}(6.0$ $\mathrm{mmol}$ )氢化钠 (50\%, 分散在矿物油中)中的矿物油, 加 入 $20 \mathrm{~mL}$ 无水 $\mathrm{DMF}$ 及 $1.7 \mathrm{~g}(5.0 \mathrm{mmol}) \mathrm{L} 1$, 加热升温 至 $100^{\circ} \mathrm{C}$, 有大量气体放出, 溶液颜色从浅黄变为 棕黄. 然后加入 $1.1 \mathrm{~g}(10 \mathrm{mmol})$ 溴乙烷, 继续反应 $12 \mathrm{~h}$. 过滤去除 $\mathrm{NaBr}$, 在滤液中加入大量乙醚, 得黄色固 体. 粗品用硅胶柱层析分离, $\mathrm{CH}_{2} \mathrm{Cl}_{2} / \mathrm{CH}_{3} \mathrm{OH}(15: 1$, $\mathrm{v} / \mathrm{v})$ 作洗脱剂, 产品再在乙醇中重结晶, 得白色固体, 产率 55\%, 元素分析测定值(\%): C, 77.47, H, 4.91, N, 17.08; $\mathrm{C}_{21} \mathrm{H}_{16} \mathrm{~N}_{4}$ 计算值(\%): C, 77.75, H, 4.93, N, 17.27; ${ }^{1} \mathrm{H} \operatorname{NMR}\left(\mathrm{CD}_{3} \mathrm{Cl}\right.$ 中) $\delta: 9.20(\mathrm{~d}, 2 \mathrm{H}), 9.19(\mathrm{~d}, 1 \mathrm{H}), 8.64(\mathrm{~d}$, $1 \mathrm{H}), 7.77(\mathrm{~m}, 4 \mathrm{H}), 7.73(\mathrm{~m}, 3 \mathrm{H}), 4.67(\mathrm{q}, 2 \mathrm{H}), 1.64 \mathrm{ppm}$ $(\mathrm{m}, 3 \mathrm{H})$.

(v) 三(二苯甲酰甲烷基))(3-乙基-2-苯基咪唑 [4, 5-f]1, 10-菲洛琳)合铕(III) $\left[\mathrm{Eu}(\mathrm{DBM})_{3} \mathbf{L 2}\right]$ 将等摩 尔的 $\mathrm{Eu}(\mathrm{DBM})_{3} \cdot 2 \mathrm{H}_{2} \mathrm{O}(0.85 \mathrm{~g}, 1.0 \mathrm{mmol})$ 和配体 L2 $(0.32 \mathrm{~g}, 1.0 \mathrm{mmol})$ 在 $25 \mathrm{~mL}$ 乙醇中回流 $2 \mathrm{~h}$, 得黄色 固体，抽滤，固体用少量乙醇洗涤，烘干. 产率 $85 \%$, 元素分析测定值(\%): C $68.98, \mathrm{H}, 4.19, \mathrm{~N}, 5.12$; $\mathrm{C}_{66} \mathrm{H}_{49} \mathrm{~N}_{4} \mathrm{O}_{6} \mathrm{Eu}$ 计算值(\%): C, 69.17, H, 4.31, N, 4.89.

(vi) $\mathrm{N}$-(3-溴丙基)咔唑(Nbpc) 搅拌下, 温度 为 $50 \sim 55^{\circ} \mathrm{C}$ 时, 将咔唑 $(16.7 \mathrm{~g}, 0.10 \mathrm{~mol})$ 慢慢加入到 1 , 3 -二溴丙烷 $(30.3 \mathrm{~g}, 0.15 \mathrm{mmol}$ )、饱和 $\mathrm{NaOH}$ 溶液 (50 $\mathrm{mL})$ 、四丁基溴化铵 $(0.40 \mathrm{~g})$ 和甲苯 $(60 \mathrm{~mL})$ 的混合体 系中(大约持续 $4 \mathrm{~h}$ ). 继续在同样温度下搅拌反应 $6 \mathrm{~h}$ 后, 分出有机相, 用去离子水洗涤, 无水硫酸钠干燥, 旋蒸除去溶剂得粗产品. 粗品用硅胶柱层析分离, $\mathrm{CH}_{2} \mathrm{Cl}_{2}$-石油醚 $\left(30 \sim 60^{\circ} \mathrm{C}\right)(1: 4, \mathrm{v} / \mathrm{v})$ 作洗脱剂, 得白 色固体，产率 $68 \% .{ }^{1} \mathrm{H} \mathrm{NMR}\left(\mathrm{CD}_{3} \mathrm{Cl}\right.$ 中) $\delta: 8.11(\mathrm{~d}, 2 \mathrm{H})$, 7.49(t, 2H), 7.45(d, 2H), 7.26(t, 2H), 4.38(t, 2H), 3.40(t, $2 \mathrm{H}), 2.11(\mathrm{~m}, 2 \mathrm{H})$.

(vii) 2-苯基-3-[3-(咔唑基-9-)丙基]咪唑[4，5-f]1， 10-菲洛琳(L3) 方法同 $\mathbf{L 2}$ 的合成, 用 $\mathrm{Nbpc}$ 代替 溴乙烷. ${ }^{1} \mathrm{H} \mathrm{NMR}\left(\mathrm{CD}_{3} \mathrm{Cl}\right.$ 中 $) \delta: 9.11(\mathrm{q}, 1 \mathrm{H}), 8.99(\mathrm{q}, 1 \mathrm{H})$, 8.88(q, $1 \mathrm{H}), 8.20(\mathrm{~d}, 2 \mathrm{H}), 7.66(\mathrm{~m}, 3 \mathrm{H}), 7.55(\mathrm{~m}, 3 \mathrm{H})$, 7.44(m, 3H), 7.33(m, 2H), 7.28(m, 2H), 6.55(t, $1 \mathrm{H})$, 4.52(t, 2H), 4.44(t, 2H), 2.00(m, mH).

(viii) 三(二苯甲酰甲烷基))\{2-苯基-3-[3-(咔唑基9-) 丙基] 咪唑 [4, 5-f]1，10-菲洛琳 \} 合铕 ( III ) $\left[\mathrm{Eu}(\mathrm{DBM})_{3} \mathbf{L 3}\right]$ 方法同 $\mathrm{Eu}(\mathrm{DBM})_{3} \mathrm{~L} 2$ 的合成，用 L3 代替 L2. 元素分析测定值(\%): C, 71.10, H, 4.59, N, 5.13; $\mathrm{C}_{79} \mathrm{H}_{58} \mathrm{~N}_{5} \mathrm{O}_{6} \mathrm{Eu}$ 计算值(\%): C, 71.61, H, 4.41, N, 5.28 .

\section{2 结果和讨论}

\section{1 吸收光谱}

图 3 是配体 $\mathrm{L} N(N=1 \sim 3)$ 和 $\mathrm{Nbpc}$ 紫外可见光谱. 配体 L1 的 $\pi-\pi$ *跃迁所产生的吸收峰位于 275,289 和 $317 \mathrm{~nm}$ 处, 而在 L2 的紫外可见光谱中, 与 L1 相比, 前两个吸收峰稍有蓝移, 最后一个长波长处的吸收 峰几乎消失, 其原因可能是配体 L1 比 L2 共轭程度大. 当 L1 中的咪唑环上的氢被乙基取代后生成 L2，由于 


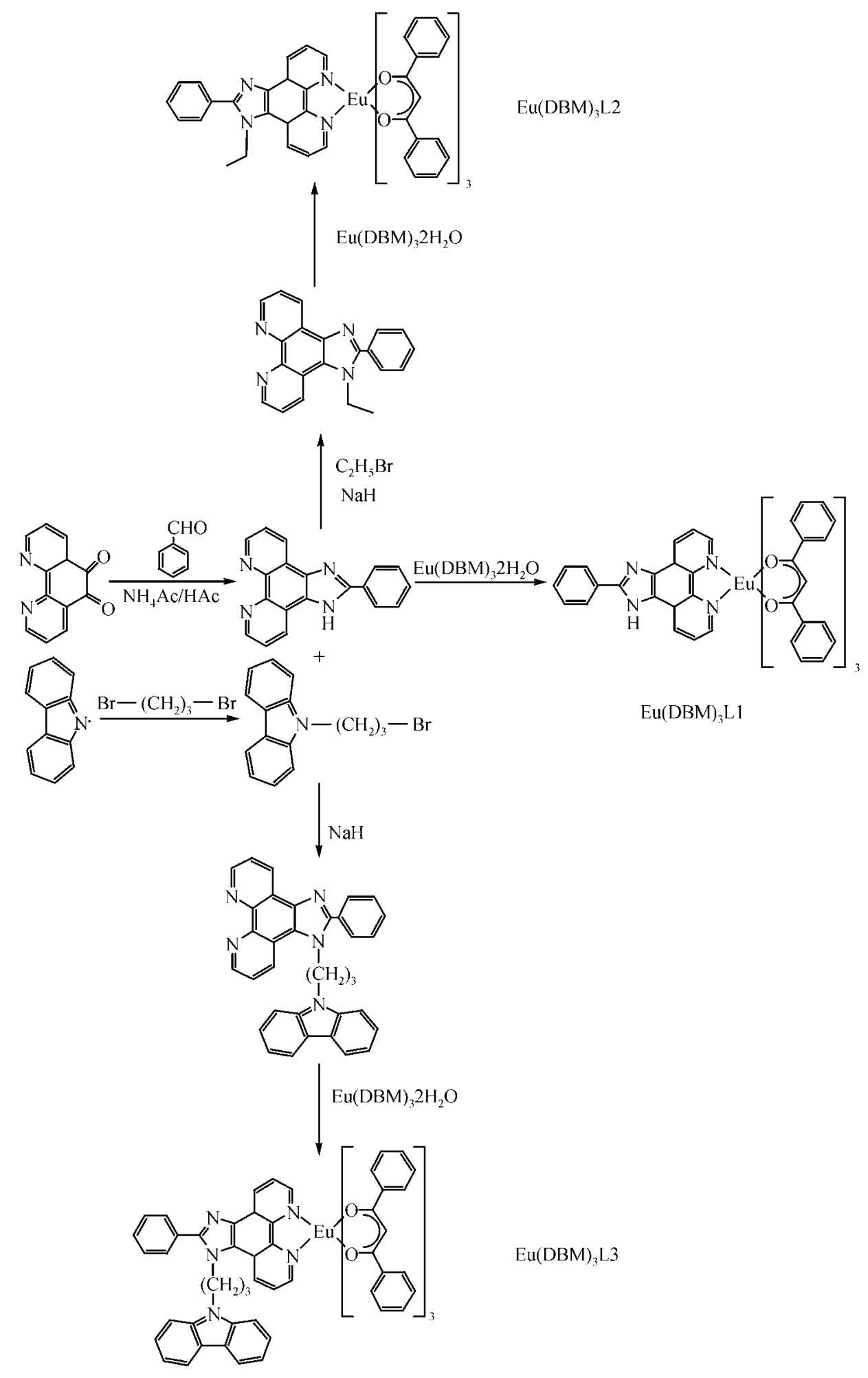

图 2 配体及相应配合物的合成路线 


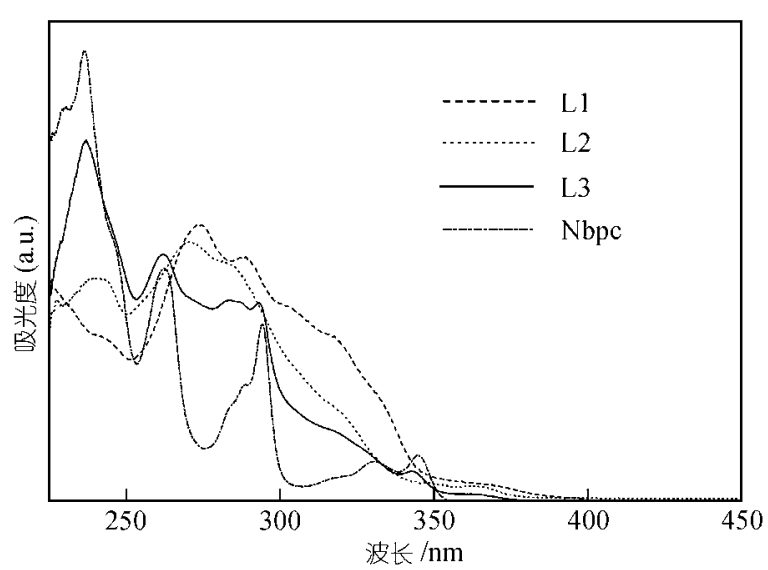

图 3 配体 $\mathrm{L} N(N=1 \sim 3$ )和 $\mathrm{Nbpc}$ (THF 中)的紫外可见光谱

空间位阻，导致后者的共轭程度减小. 比较 L3 与 $\mathrm{Nbpc}$ 及 L2 的紫外可见吸收光谱, 很显然, 在 L3 的吸 收光谱中, 262, 293 和 $344 \mathrm{~nm}$ 处吸收峰的出现证明配 体 L3 中咔唑基团的存在. $\mathrm{Eu}(\mathrm{DBM})_{3} \mathrm{~L} N(N=1 \sim 3)$ 及 $\mathrm{Eu}(\mathrm{DBM})_{3} \cdot 2 \mathrm{H}_{2} \mathrm{O}$ 的紫外吸收光谱(在 THF 中)很相似, DBM 的 $\pi-\pi *$ 跃迁吸收 $(251,280$ 和 $355 \mathrm{~nm})$ 在配合物 中占主导地位, 使得中性配体的吸收特征被掩盖. 此 外, 配合物的 $\varepsilon$ 值大于 $\mathrm{Eu}(\mathrm{DBM})_{3} \cdot 2 \mathrm{H}_{2} \mathrm{O}$ 和相应配体 的 $\varepsilon$ 值之和, 同样说明三元配合物的形成.

\section{2 苂光光谱及热稳定性}

三个配合物的固体粉末及在四氢呋喃溶液中的 光致发光光谱基本相似(以配合物 $\mathrm{Eu}(\mathrm{DBM})_{3} \mathrm{~L} 3$ 为例, 如图 4), 显示稀土 $\mathrm{Eu}(\mathrm{III})$ 离子的特征发射, 没有配体 的苂光, 其发射峰的位置为 $580,598,613$ 和 $653 \mathrm{~nm}$, 它们分别对应于 $\mathrm{Eu}(\mathrm{III})$ 离子的 ${ }^{5} \mathrm{D}_{0} \rightarrow{ }^{7} \mathrm{~F}_{0},{ }^{5} \mathrm{D}_{0} \rightarrow{ }^{7} \mathrm{~F}_{1},{ }^{5} \mathrm{D}_{0}$ $\rightarrow{ }^{7} \mathrm{~F}_{2}$ 和 ${ }^{5} \mathrm{D}_{0} \rightarrow{ }^{7} \mathrm{~F}_{3}$ 的特征跃迁, 其中以 ${ }^{5} \mathrm{D}_{0} \rightarrow{ }^{7} \mathrm{~F}_{2}$ 的发射 为最强. 它们的 ${ }^{5} \mathrm{D}_{0} \rightarrow{ }^{7} \mathrm{~F}_{2}$ 跃迁与 ${ }^{5} \mathrm{D}_{0} \rightarrow{ }^{7} \mathrm{~F}_{1}$ 跃迁谱线的 相对强度比都较大 $(>10)$, 表现出很好的单色性. 配 合物的荧光强度与所配位的中性配体有关. 在溶液 中, $\mathrm{Eu}(\mathrm{DBM})_{3} \mathrm{~L} 1, \mathrm{Eu}(\mathrm{DBM})_{3} \mathrm{~L} 2$ 和 $\mathrm{Eu}(\mathrm{DBM})_{3} \mathrm{~L} 3$ 的光 致发光量子效率分别为 $9.98,0.98$ 和 $11.02 \%$, 都比 $\mathrm{Eu}(\mathrm{DBM})_{3} \cdot 2 \mathrm{H}_{2} \mathrm{O}$ (其光致发光量子效率为 $4.2 \%$ )要大 许多, 这说明中性配体取代配位水分子以后大大减 少了水中高能 $\mathrm{O}-\mathrm{H}$ 振动所带来的能量损失, 有效提 高了配合物的量子效率. 不仅配合物 $\mathrm{Eu}(\mathrm{DBM})_{3} \mathrm{~L} 2$ 和 $\mathrm{Eu}(\mathrm{DBM})_{3} \mathrm{~L} 3$ 的溶液光致发光量子效率比

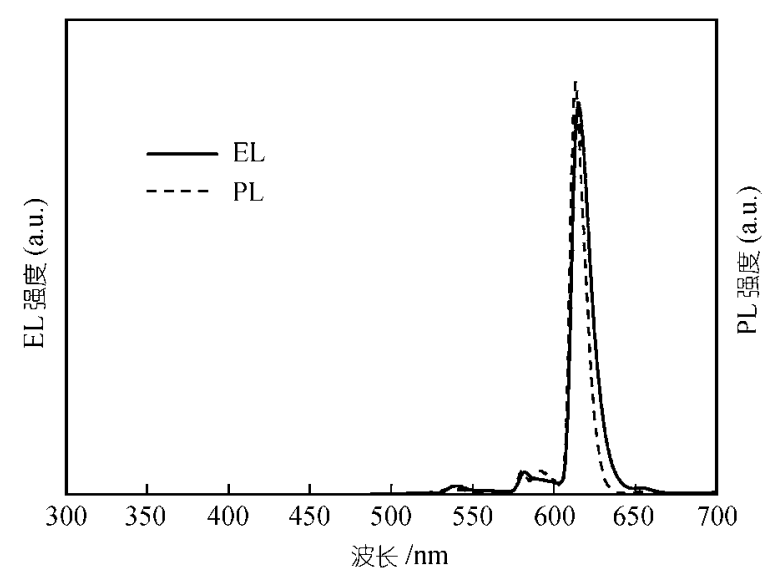

图 $4 \mathrm{Eu}(\mathrm{DBM})_{3} \mathrm{~L} 3(60 \mathrm{~nm}$ 左右薄膜, 在石英基片上)的光 致发光光谱 $\left(\mathrm{PL}\right.$, 右) $\left(\lambda_{\mathrm{ex}}=388 \mathrm{~nm}\right.$ ) 和器件 $\mathrm{A} 3$ 在 $16 \mathrm{~V}$ 直流 电压驱动下的电致发光光谱 $(\mathrm{EL}$, 左)

$\mathrm{Eu}(\mathrm{DBM})_{3} \mathrm{~L} 1$ 的大，它们的固体荧光强度也是 $\mathrm{Eu}(\mathrm{DBM})_{3} \mathrm{~L} 1$ 的 5 倍之多. 可能的原因有两个. 一是 配体L1 中存在高能的 N-H振动, 对荧光有淬灭作用, 而在配体L2 或 L3 中, 乙基或含咔唑的丙基取代了 $\mathrm{N}-\mathrm{H}$ 上的 $\mathrm{H}$ ，消除了其影响; 二是配体L2 或L3 比L1 更能有效地将所吸收的能量传递给 $\mathrm{Eu}(\mathrm{III})^{[18]}$.

热重和差热分析表明 $\mathrm{Eu}(\mathrm{DBM})_{3} \mathrm{~L} 1$ 和 $\mathrm{Eu}$ $(\mathrm{DBM})_{3} \mathrm{~L} 2$ 在分解前无熔点, 而 $\mathrm{Eu}(\mathrm{DBM})_{3} \mathrm{~L} 3$ 的熔点 为 $282^{\circ} \mathrm{C} . \mathrm{Eu}(\mathrm{DBM})_{3} \mathrm{~L} 1, \mathrm{Eu}(\mathrm{DBM})_{3} \mathrm{~L} 2$ 和 $\mathrm{Eu}(\mathrm{DBM})_{3} \mathrm{~L} 3$ 的起始分解温度分别为 358,381 和 $408^{\circ} \mathrm{C}$, 表明 $\mathrm{Eu}(\mathrm{DBM})_{3} \mathrm{~L} 3$ 的热稳定性最好, 其次为 $\mathrm{Eu}(\mathrm{DBM})_{3} \mathrm{~L} 2$.

\section{3 电致发光性质}

为了比较配合物的电致发光性质, 我们以 ITO 为阳极, $\mathrm{Mg}_{0.9} \mathrm{Ag}_{0.1}$ 作为阴极, TPD 作空穴传输层, 配 合物 $\operatorname{Eu}(\mathrm{DBM})_{3} \mathrm{~L} N(N=1 \sim 3)$ 作为发光层和电子传输 层, 制作了双层结构的电致发光器件 ITO/TPD $(50$ $\mathrm{nm}) / \mathrm{Eu}(\mathrm{DBM})_{3} \mathrm{~L} N(N=1 \sim 3)(50 \mathrm{~nm}) / \mathrm{Mg}: \operatorname{Ag}(200 \mathrm{~nm}) /$ $\mathrm{Ag}(100 \mathrm{~nm})$, 以器件 $\mathrm{A} 1 \sim 3$ 分别代表配合物 $\mathrm{Eu}$ $(\mathrm{DBM})_{3} \mathrm{~L} 1, \mathrm{Eu}(\mathrm{DBM})_{3} \mathrm{~L} 2$ 和 $\mathrm{Eu}(\mathrm{DBM})_{3} \mathrm{~L} 3$ 所制作的器 件. 在直流电压驱动下, 所有器件均能发出 $\mathrm{Eu}(\mathrm{III})$ 离 子的特征苂光. 如图 4 所示, 其电致发光的光谱与光 致发光光谱基本相同.

器件 $\mathrm{A} 1 \sim \mathrm{A} 3$ 的最大亮度分别为 $3.7 \mathrm{~cd} / \mathrm{m}^{2}(17 \mathrm{~V})$, $158 \mathrm{~cd} / \mathrm{m}^{2}(20 \mathrm{~V})$ 和 $561 \mathrm{~cd} / \mathrm{m}^{2}(16 \mathrm{~V})$ (见表 1). 很明显, 
比较而言, 由于 $\mathrm{Eu}(\mathrm{DBM})_{3} \mathrm{~L} 1$ 较差的光致发光性能及 热稳定性，因此其电致发光性能也最差. 在三个配合 物中, $\mathrm{Eu}(\mathrm{DBM})_{3} \mathrm{~L} 3$ 不仅具有与 $\mathrm{Eu}(\mathrm{DBM})_{3} \mathrm{~L} 2$ 相近的 光致发光效率，以及最好的热稳定性，而且由于咔唑 基团的引入，使其具有良好的空穴传输性能. 由 $\mathrm{Eu}(\mathrm{DBM})_{3} \mathrm{~L} 3$ 所制作的双层器件最大亮度达 561 $\mathrm{cd} / \mathrm{m}^{2}$, 是文献报道的铕配合物制备的双层器件中结 果最好的之一 ${ }^{[19]}$. 器件 $\mathrm{A} 2$ 与器件 $\mathrm{A} 3$ 的电流-电压曲 线如图 5 所示.

表 1 材料的分子结构、光致发光效率以及热稳定性 对其电致发光性质的影响

\begin{tabular}{lccc}
\hline \multicolumn{1}{c}{ 配合物 } & $\mathrm{Eu}(\mathrm{DBM})_{3} \mathrm{~L} 1$ & $\mathrm{Eu}(\mathrm{DBM})_{3} \mathrm{~L} 2$ & $\mathrm{Eu}(\mathrm{DBM})_{3} \mathrm{~L} 3$ \\
\hline 中性配体 & $\mathrm{L} 1$ & $\mathrm{~L} 2$ & $\mathrm{~L} 3$ \\
热分解温度 & $358^{\circ} \mathrm{C}$ & $381^{\circ} \mathrm{C}$ & $408^{\circ} \mathrm{C}$ \\
相对苂光强度(固体) & 1 & 5.3 & 5.2 \\
$\mathrm{PL}$ 效率(THF 中) & $9.98 \%$ & $10.98 \%$ & $11.02 \%$ \\
$\mathrm{EL}$ 最大亮度 & $3.7(17 \mathrm{~V})$ & $197(20 \mathrm{~V})$ & $561(16 \mathrm{~V})$ \\
\hline
\end{tabular}

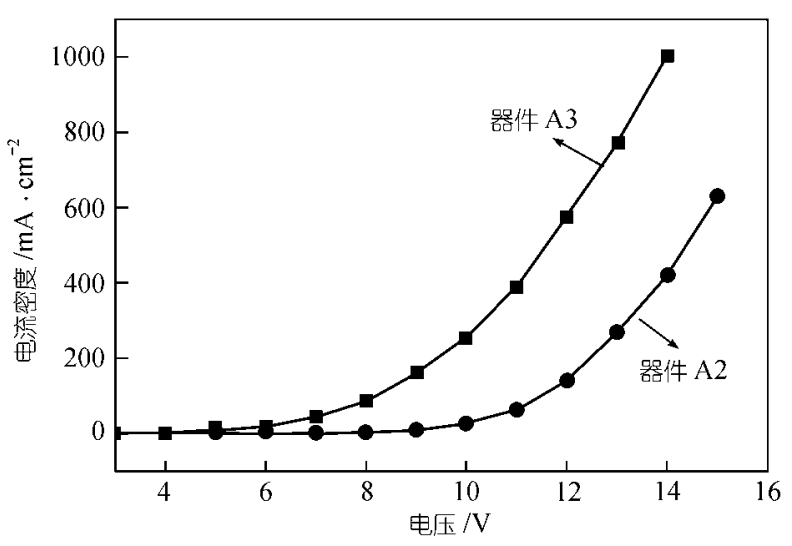

图 5 器件 $\mathrm{A} 2$ 与器件 $\mathrm{A} 3$ 的电流-电压曲线

分别将配合物 $\mathrm{Eu}(\mathrm{DBM})_{3} \mathrm{~L} 2$ 或 $\mathrm{Eu}(\mathrm{DBM})_{3} \mathrm{~L} 3$ 掺杂 在 TPD 中作为发光层, TPD 和 AlQ 分别作为空穴传 输层和电子传输层, 制作了器件 $\mathrm{ITO} / \mathrm{TPD}(30 \mathrm{~nm}) /$ TPD : $\mathrm{Eu}(\mathrm{DBM})_{3} \mathrm{~L} 2\left(\right.$ 或 $\left.\mathrm{Eu}(\mathrm{DBM})_{3} \mathrm{~L} 3\right)(1: 2, \mathrm{~mol}$ : $\mathrm{mol})(30 \mathrm{~nm}) / \mathrm{Eu}(\mathrm{DBM})_{3} \mathrm{~L} 2(20 \mathrm{~nm}) / \mathrm{AlQ}(20 \mathrm{~nm}) / \mathrm{Mg}_{0.9}$ $\mathrm{Ag}_{0.1}(200 \mathrm{~nm}) / \mathrm{Ag}(100 \mathrm{~nm}$ )(分别用器件 B2 和器件 B3 表示配合物 $\mathrm{Eu}(\mathrm{DBM})_{3} \mathrm{~L} 2$ 和 $\mathrm{Eu}(\mathrm{DBM})_{3} \mathrm{~L} 3$ 所制作的器 件). 器件 B2 的电致发光光谱与其光致发光光谱一 致, 而且其最大亮度比其相应的双层器件 A2 高, 达 到 $350 \mathrm{~cd} / \mathrm{m}^{2}(16 \mathrm{~V})$. 显然, 掺杂后由于发光层的空穴
传输性能的改善提高了器件的电致发光性能.

但是，器件 B3 的电致发光光谱中出现 AlQ 的发 射，导致其不再是纯的红光. 这是因为配合物 $\mathrm{Eu}(\mathrm{DBM})_{3} \mathrm{~L} 3$ 具有较好的空穴传输性能，掺杂在空穴 传输材料 TPD 中是不合适的. 为了得到纯铕的特征 发射, 我们引入了具有很好电子传输性质的 $\mathrm{OXD}$, 制成器件 C3: ITO/TPD $(25 \mathrm{~nm}) /\left[\mathrm{Eu}(\mathrm{DBM})_{3} \mathrm{~L} 3(10 \mathrm{~nm})\right.$ : $\operatorname{OXD}(5 \mathrm{~nm})]_{4} / \mathrm{OXD}(5 \mathrm{~nm}) / \operatorname{Alq}(15 \mathrm{~nm}) / \mathrm{Mg}_{0.9} \mathrm{Ag}_{0.1}(200$ $\mathrm{nm}) / \mathrm{Ag}(100 \mathrm{~nm})$. 正如所希望的，载流子的复合区域 很好地限制在发光层 $\mathrm{Eu}(\mathrm{DBM})_{3} \mathrm{~L} 3$ 中 (见图 6, 不同电 压下器件 $\mathrm{C} 3$ 的电致发光光谱), 器件最大亮度高达 $770 \mathrm{~cd} / \mathrm{m}^{2}(18 \mathrm{~V}, 0.05 \mathrm{Lm} / \mathrm{W})$, 最大流明效率为 0.36 $\mathrm{Lm} / \mathrm{W}\left(5.9 \mathrm{~cd} / \mathrm{m}^{2}, 10 \mathrm{~V}\right)$ (图 7). 特别值得提出的是, 器 件 C3 的电致发光光谱中, 没有来自 AlQ 的发射.

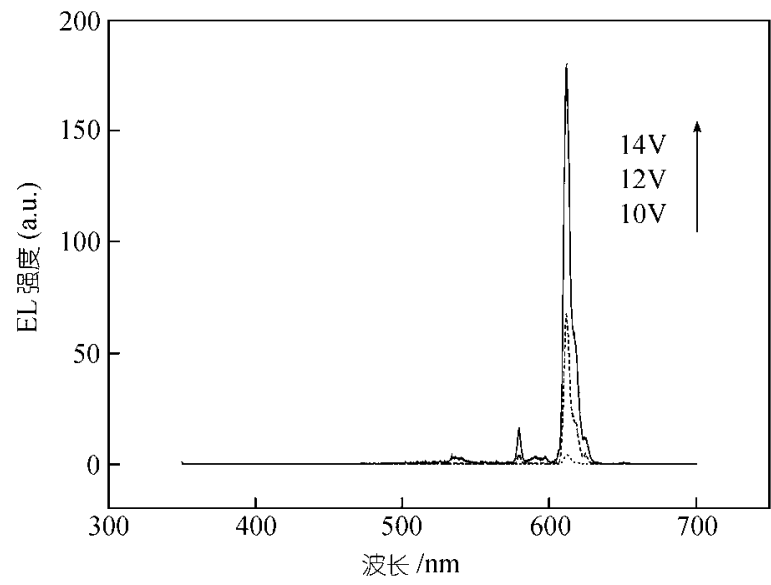

图 6 不同电压下器件 $\mathrm{C} 3$ 的电致发光光谱

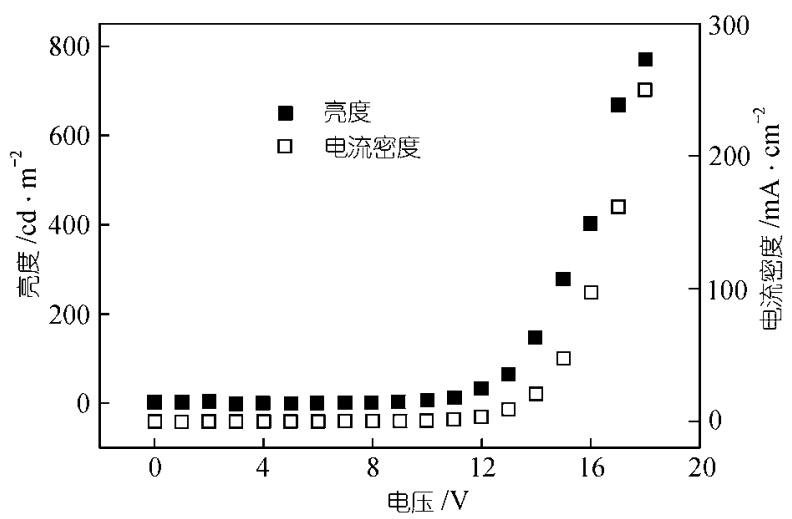

图 7 器件 $\mathrm{C} 3$ 亮度-电流密度-电压图和 $\mathrm{EL}$ 效率-电压图 
此外, 用 BCP 代替 OXD 所制作的器件 D3, ITO/ $\operatorname{TPD}(50 \mathrm{~nm}) /\left[\mathrm{Eu}(\mathrm{DBM})_{3} \mathrm{~L} 3(5 \mathrm{~nm}): \mathrm{BCP}(5 \mathrm{~nm})\right]_{4} / \mathrm{BCP}$ $(20 \mathrm{~nm}) / \operatorname{AlQ}(10 \mathrm{~nm}) / \mathrm{Mg}_{0.9} \mathrm{Ag}_{0.1}(200 \mathrm{~nm}) / \operatorname{Ag}(100 \mathrm{~nm})$, 其最大亮度在 $18 \mathrm{~V}$ 时高达 $1419 \mathrm{~cd} / \mathrm{m}^{2}$, 且为纯的铕的 特征发射, 最大流明效率为 $0.88 \mathrm{Lm} / \mathrm{W}\left(6 \mathrm{~V}, 7.2 \mathrm{~cd} / \mathrm{m}^{2}\right)$. 图 8 和 9 分别是器件 D3 的亮度-电流密度-电压图和 $\mathrm{EL}$ 效率-电压图. 从图中可以看出，随着电压的升高， 电流密度逐渐增大，亮度也随着增大，器件 D3 的启 动电压仅为 $5 \mathrm{~V}$ (启动电压的定义为亮度达 $1 \mathrm{~cd} / \mathrm{m}^{2}$ 时 的电压), 在低电流密度区域, 随着电压升高, 器件的 效率也增大. 电压为 $6 \mathrm{~V}$ 时, 效率达到了最大值, 当 电压继续增加，电流密度和亮度都迅速增加，但效率 却下降. 这是因为随着电压升高, 电流密度增大, 注 入的空穴电子对增多, 激子数也相应增多, 但由于发 射子饱和，引起激子湮灭，导致效率下降.

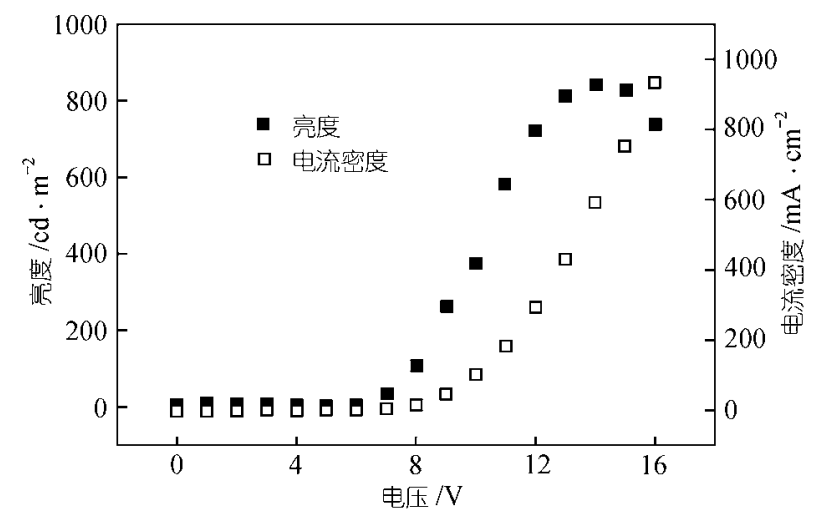

图 8 器件 D3 亮度-电流密度-电压图和 EL 效率-电压图

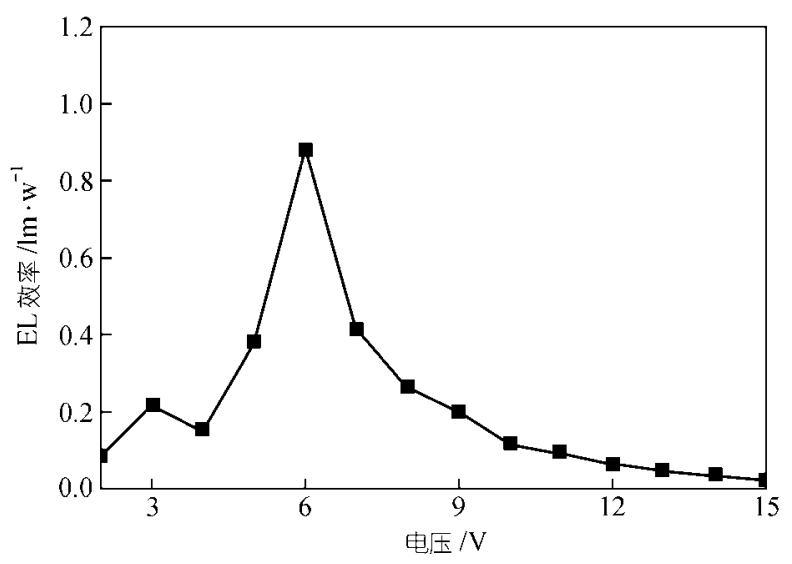

图 9 器件 D3 的 $\mathrm{EL}$ 效率-电压图

\section{3 结论}

实验证明，对中性配体的适当修饰，可有效改善 配合物材料的光致发光性质、热稳定性和成膜性能, 从而提高其器件的电致发光的亮度和效率. 双层器 件 ITO/TPD(50 nm)/Eu(DBM $)_{3} \mathrm{~L} 3(50 \mathrm{~nm}) / \mathrm{Mg}_{0.9} \mathrm{Ag}_{0.1}$ $(200 \mathrm{~nm}) / \mathrm{Ag}(100 \mathrm{~nm})$ 的最大亮度达到 $561 \mathrm{~cd} / \mathrm{m}^{2}(16$ $\mathrm{V})$, 四层器件 $\mathrm{TPD}(50 \mathrm{~nm}) /\left[\mathrm{Eu}(\mathrm{DBM})_{3} \mathrm{~L} 3(5 \mathrm{~nm}): \mathrm{BCP}\right.$ $(5 \mathrm{~nm})]_{4} / \mathrm{BCP}(20 \mathrm{~nm}) / \mathrm{AlQ}(10 \mathrm{~nm}) / \mathrm{Mg}_{0.9} \mathrm{Ag}_{0.1}(200 \mathrm{~nm}) /$ $\mathrm{Ag}(100 \mathrm{~nm})$ 最大亮度达到 $1491 \mathrm{~cd} / \mathrm{m}^{2}(18 \mathrm{~V})$, 都为纯 的铕的特征发射. 相信经过进一步器件结构的优化, $\mathrm{Eu}(\mathrm{DBM})_{3} \mathrm{~L} 3$ 电致发光器件的性能能够得到更好的改 善. 该配合物是一种有实际应用前景的纯红色电致 发光材料.

\section{参考文献}

1 Sun P P, Duan J P, Shih H T, et al. Europium complex as a highly efficient red emitter in electroluminescent devices. Appl Phys Lett, 2002, 81: 792 793[DOI]

2 Heil H, Steiger J, SchmechelR,et al. Tri(dibenzolymethane)(monophenanthroline)europium(III) based red emitting organic light emitting diodes. J Appl Phys, 2001, 90: 5357 5362[DOI]

3 Liang C L, Zhao D, Hong Z R, et al. Improved performance of electroluminescent devices based on an europium complex. Appl Phys Lett, 2000, 76, 67 69[DOI]

4 Kido J, Hayase H, Honggawa K, et al. Bright red light-emitting organic electroluminescent devices having a europium complex as an emitter. Appl Phys Lett, 1994, 65: 2124 2126[DOI]

5 Mitsuharu N, Kazutaka I, Masanao E. Efficient red electroluminescence from new europium complex. Chem Lett, 2001: 320 321

6 Kido J, Nagai K, Okamoto Y. Organic electroluminescent devices using lanthanide complexes. J Alloys Compd, 1993, 192: 30 33 [DOI]

7 Wang J, Wang R, Yang J, et al. First oxadiazole-functionalized terbium(III) $\beta$-diketonate for organic electroluminescence. J Am Chem Soc, 2001, 123: 6179 6180[DOI]

8 Capecchi S, Renault O, Moon D G.. High-efficiency organic electroluminescent devices using an organoterbium emitter. Adv Mater, 2000, 12: 1591 1594[DOI]

9 Huang L, Wang K Z, Huang C H, et al. Synthetic and electroluminescent properties of two novel europium complexes with benzimidazole derivatives as second ligands. Synth Met, 2002, 128: 241 245

10 Huang L, Wang $\mathrm{K} \mathrm{Z}$, Huang $\mathrm{C} \mathrm{H}$, et al. Bright red electroluminescent devices using novel second-ligand-contained europium complexes as emitting layers. J Mater Chem, 2001, 11: 790 $793[\mathrm{DOI}]$ 
11 Hu W P, Matsumura M, Wang M Z, et al. Efficient red electroluminescence from devices having multilayers of a europium complex. Appl Phys Lett, 2000, 77: 4271 4273[DOI]

12 Yu G., Liu Y Q, Wu X, et al. Soluble Europium complexes for Light-Emitting diodes chem. Mater, 2000, 12: 2537 2541[DOI]

13 Gao X C, Cao H, Huang C H, et al. Electroluminescence of a novel terbium complex. Appl Phys Lett, 1998, 72: 2217 2219[DOI]

14 Archer D, Chen H, Thompson L C. Synthesis, characterization, and luminescence of europium(III) schiff base complexes. Inorg Chem, 1998, 37: 2089 2095[DOI]

15 Haas Y, Stein G. Pathways of radiative and radiationless transitions in europium (III) solutions: Role of solvents and anions. J
Phys Chem, 1971, 75: 3668 3677

16 Hiort $\mathrm{C}$, Lincoln $\mathrm{P}$, Norden B. DNA binding of $\Delta$ - and $\Lambda$ $\left[\mathrm{Ru}(\text { phen })_{2} \mathrm{DPPZ}\right]^{2+}$. J Am Chem Soc, 1993, 115: 3448 3454

17 Steck E A, Day A R. Reactions of Phenanthraquinone and retenequinone with aldehydes and ammonium acetate in acetic acid solution. J Am Chem Soc, 1943, 65: 452 456

18 Bian Z Q, Wang K Z, Jin L P. Syntheses, spectroscopic and crystal structural studies of novel imidazo [4,5-f]1,10-phenanthroline derivatives and their $\mathrm{Eu}(\mathrm{III})$ ternary complexes with dibenzoylmethane. Polyhedron, 2002, 21 (3): 313 319[DOI]

19 Guan M, Bian Z Q, Xin H, et al. Bright red light-emitting electroluminescence devices based on a novel carbazole-functionalized europium complex. New J Chem, 2003, 27: 1731 1734[DOI] 